\title{
Biomarkers of the Caseous Lymphadenitis in Sheep by NMR-Based Metabolomics
}

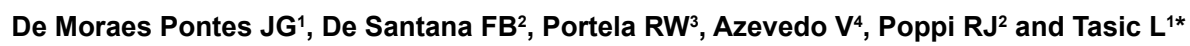

${ }^{1}$ Department of Organic Chemistry, Institute of Chemistry, State University of Campinas, Brazil

${ }^{2}$ Department of Analytical Chemistry, Institute of Chemistry, State University of Campinas, Brazil

${ }^{3}$ Laboratory of Immunology and Molecular Biology, Institute of Health Sciences, Federal University, Bahia, Brazil

${ }^{4}$ Department of General Biology, Institute of Biological Sciences, Federal University of Minas Gerais, Belo Horizonte, Brazil

\begin{abstract}
Caseous Lymphadenitis (CLA) is a disease that affects animals, such as sheep, whose etiologic agent is Corynebacterium pseudotuberculosis. In sheep, it causes enormous damages, expressed through a reduction in milk, meat and wool production, or death of infected animals, and, also, difficulties in sterilizing infected animals habitats. Another problem in the control of this disease is its late diagnosis. Thus, we present the pioneer results on NMRbased metabolomics applied in CLA with the aim to detect blood serum profile alterations provoked by a pathogen in C. pseudotuberculosis seropositive sheep. We have compared two types of serum samples: The ones taken from the seropositive animals, which we called infected, and others taken from the seronegative (healthy) animals. Significant metabolomics profile changes occurred in the spectral regions $\delta: 0.20$ to $2.20 ; 3.20$ to 4.40 and 6.40 to 8.00 that refer to hydrogen atoms of proteins, organic acids, alcohols, lipids and some amino acids, correspondingly. When applying chemometrics, a significative separation of 59 serum samples into two groups, sick and healthy animals, was achieved. Additionally, key-metabolites that were only present in the group of sick animals and that can be considered as exclusively bacteria derived were pointed as possible biomarkers for CLA. Therefore, these data might contribute to the development of a non-invasive NMR-based diagnostic method, as well as to bring new insights into the development of new immunoprohylaxis tools.
\end{abstract}

Keywords: Biomarkers; Caseous lymphadenitis; Chemometrics; Metabolomics; Nuclear Magnetic Resonance (NMR)

Abbreviations: BMRB: Biological Magnetic Resonance Data Bank; CLA: Caseous Lymphadenitis; CPMG: Carr-Purcell-Meiboom-Gill; FID: Free Induction Decay; FNR: False Negative Rate; FPR: False Positive Rate; HSQC: Heteronuclear Single Quantum Coherence; LB: Line Broadening; NMR: Nuclear Magnetic Resonance; PC: Principal Component; PCA: Principal Component Analysis; PCR: Polymerase Chain Reaction; PLS-DA: Partial Least Squares Discriminant Analysis; PRIMe: Platform for RIken Metabolomics; RMSEC: Root Mean Square Error of Calibration; RMSECV: Root Mean Square Error of CrossValidation; ROC: Receiver Operating Characteristic; SNV: Standard Normal Variate; TBI: Triple Resonance Broadband Inverse; TN: True Negative; TP: True Positive; VIP: Variable Importance in Projection; WATERGATE: Water Suppression by Gradient Tailored Excitation

\section{Introduction}

Corynebacterium pseudotuberculosis is a Gram-positive bacterium that infects mainly sheep and goats causing Caseous Lymphadenitis (CLA). However, it is also able to infect pigs, horses (causing the disease called ulcerative lymphangitis), cattle, buffaloes, Patagonian Huemul (Hippocamelus bisulcus) and even humans [1-8]. Transmission of CLA in sheep may occur during some procedures such as shear or castration using infected tools, as well as through the penetration of the bacteria in skin lesions [1-9]. Another factor that contributes to the dissemination of this disease is the confinement of animals in corrals and barns, once C. pseudotuberculosis is persistent and survives in these environments for an extended period [10].

CLA is characterized by lymphoadenomegaly and increases of vascular permeability, facilitating other ways of infections in the sheep. After the incubation period of the pathogen (1 to 3 months), the formation of encapsulated purulent abscesses with characteristic color occurs. The direct contact with these abscesses is also one form of transmission of disease [11].
C. pseudotuberculosis is a pathogen of worldwide incidence. There are reports of its occurrence in several countries such as South Africa, Argentina, Australia, Brazil, United States, European countries and many others [11-17].

CLA is responsible for significant economic losses in agribusiness due to the reduction of meat, milk, and wool production. Because there is no effective treatment for the disease, it is recommended that infected animals should be euthanized, as a measure of the control of illness $[9,18]$. The use of antibiotics or antimicrobial chemotherapy for the treatment of C. pseudotuberculosis is not efficient because the bacteria remain viable inside encapsulated granulomatous lesions, where drugs are unable to penetrate. Also, the bacterium is resistant to several drugs such as penicillin, and some substances that are used for the control of the bacterial growth inside the abscess are toxic, such as formaldehyde [19-21].

Vaccination has been an efficient strategy for the prevention of the disease. However, vaccines are not able to prevent the dissemination of bacterium in vaccinated animals [22]. Furthermore, they do not have the same efficacy in distinct species of hosts, immediate side effects, and are not available or licensed in some countries [23].

*Corresponding author: Tasic L, Department of Organic Chemistry, Institute of Chemistry, State University of Campinas, P. O. Box 6154, Campinas, SP 13083-970, Brazil, Tel: ++ 5519 3521-1106; Fax: ++ 55193521 3023; E-mail: ljubica@iqm.unicamp.br

Received March 20, 2017; Accepted April 10, 2017; Published April 17, 2017

Citation: De Moraes Pontes JG, De Santana FB, Portela RW, Azevedo V, Poppi RJ, et al. (2017) Biomarkers of the Caseous Lymphadenitis in Sheep by NMR-Based Metabolomics. Metabolomics (Los Angel) 7: 190. doi:10.4172/2153-0769.1000190

Copyright: @ 2017 De Moraes Pontes JG, et al. This is an open-access article distributed under the terms of the Creative Commons Attribution License, which permits unrestricted use, distribution, and reproduction in any medium, provided the original author and source are credited. 
Therefore, one of the options that might ameliorate treatment of this disease is to detect CLA at the beginning of infection and intervene before the illness has spread. Thus, biomarker detection other than C. pseudotuberculosis detection through PCR (DNA, RNA) analysis is being proposed. NMR spectroscopy can be an interesting option, once it is a non-destructive and reproducible technique and a very suitable analytical platform for the identification of metabolites and biomarkers in metabolomics studies of different nature [24-29]. There are researches on C. pseudotuberculosis-hosts interactions in the scientific literature [30-33]; however, there is a lack of information on this disease's metabolomics.

In this context, the main aim of this research was to evaluate the metabolic changes in animals infected by $C$. pseudotuberculosis through the combination of NMR spectroscopy and chemometrics. Applying NMR-based metabolomics to assess the blood serum profile changes in CLA is the pioneer attempt. Our research aimed the identification of key metabolites and possible biomarkers of caseous lymphadenitis disease in sheep. We hope that the results of our research may contribute to the development of methodologies for the early diagnosis of CLA still in its asymptomatic phase, acting as a form of zoonosis control and prevention of CLA as to minimize economic losses.

\section{Materials and Methods}

\section{Samples}

Sera from 59 sheep were used in this study. 33 blood samples were taken from the animals infected with Corynebacterium pseudotuberculosis, as confirmed by bacterial isolation from caseous lesions, and further identification by biochemical [34] and molecular methods [35]. Concomitantly, sera from 26 healthy control animals were collected. The healthy sheep were picked after continuous clinical and immunological examinations, the last been characterized by $89 \%$ sensitivity and $99 \%$ specificity [36].

Animals of both genders, with the ages varying from eight months to five years, were selected. They were subjected to the same nutritional scheme and breeding procedures. Healthy sheep were bred apart and isolated from infected animals, with the objective to prevent further infections. The sample collection was obtained through vacutainer system without the use of anticoagulants. The puncture was done in the jugular vein using a $10 \mathrm{~mL}$ tube. The blood was centrifuged at 4,000 $\mathrm{rpm}$ for $10 \mathrm{~min}$, and was stored at $-80^{\circ} \mathrm{C}$ for not longer than 15 days until NMR-spectra acquisition.

\section{Nuclear magnetic resonance spectroscopy analyses}

${ }^{1} \mathrm{H}$ NMR spectra: For NMR analysis, $250 \mu \mathrm{L}$ of each animal serum sample were mixed with $250 \mu \mathrm{L}$ of deuterium oxide solvent $\left(\mathrm{D}_{2} \mathrm{O}\right.$, 99.9\%). The lactate peak ( $3 \mathrm{H}, \delta 1.33,{ }^{3} \mathrm{~J}=7 \mathrm{~Hz}$, doublet) was used as a reference [37]. The ${ }^{1} \mathrm{H}$ NMR spectra were acquired with ns $=128$ on a Bruker Avance III $600 \mathrm{MHz}$ spectrometer at $25^{\circ} \mathrm{C}$ using WATERGATE pulse sequence (p3919gp) and a TBI (Triple Resonance Broadband Inverse) probe. ${ }^{1} \mathrm{H}$ NMR spectra with $T_{2}$ filter were recorded using the pulse sequence cpmgprld-CPMG (Carr-Purcell-Meiboom-Gill) [38] also with ns $=128$.

Two-dimensional NMR spectroscopy: 2D NMR spectra were acquired using a spectrometer frequency of $600.17 \mathrm{MHz}$ for F2 and $150.91 \mathrm{MHz}$ for F1 with a free induction decay (FID) size of 2048 (F2) and 256 (F1) data points and ns=64. Edited HSQC were acquired in the spectral range between $\delta 11.73$ to -2.29 (F2) and $\delta 25.9$ to 11.0 (F1). For more details about spectra, acquisition parameters see supplementary information. HSQC contour maps were processed using $\mathrm{LB}=1.00 \mathrm{~Hz}$ (F2) and $\mathrm{LB}=0.30 \mathrm{~Hz}(\mathrm{~F} 1)$.

NMR spectra processing: After NMR-spectra recording, the data-matrix has been constructed and, then, we have tested various data preprocessing as to remove all sources of variation that were not related to our samples [39-42]. The tested preprocessing methods were: Autoscale, pareto scale, standard normal variate (SNV) and normalization by the line between 0 and 1 [43].

Chemometric analysis: PLS-DA as variation of the Partial Least Squares (PLS) regression algorithm for discriminant analysis and a supervised classification method for multivariate data, was constructed as to relate a matrix $\mathrm{X}$ (NMR data) with a matrix/vector $\mathrm{Y}$ representing the class of the sample [44,45].

In general, for PLS-DA involving 2 classes, it is common to use Class 1 and Class 0 [46]. Therefore, the Class 0 corresponds to infected sheep's blood sera, and Class 1 corresponds to healthy control samples.

In many cases, the $\mathrm{Y}$ predicted value by the model could take values close to 0 and 1 (not exactly 0 and 1). Thus, it is necessary to calculate the threshold value for separation of the classes [47]. If predicted value is lower than the specified threshold value, the sample will be considered to belong to Class 0 , and when the predicted value is higher than the threshold, the sample will be classified as a part of Class 1 . The optimal threshold value can be calculated by Bayes theorem or use the ROC curves $[43,48,49]$.

For the development of PCA and PLS-DA classification models MATLAB R2016a (Mathworks Inc.) and PLS_TOOLBOX 8.1 (Eigenvector Research) were used. Randomly, approximately twothirds of the samples were selected from healthy and infected sheep blood sera for the training set, and the remaining samples were used as the test set.

For the construction of PCA model, the optimal number of Principal Components (PC), was chosen analyzing the additive variance in X block. Moreover, for PLS-DA model the optimal number of latent variables was selected based on the Root Mean Square Error of Cross-Validation (RMSECV) in a combined analysis with the lowest difference between RMSECV and Root Mean Square Error of Calibration (RMSEC). Moreover, the additive variance in the $\mathrm{X}$ and $\mathrm{Y}$ blocks was also analyzed. Following these criteria, some models using the pre-processing already described were built. Also, the best PCA model was constructed with 4 PC. The best PLS-DA model was constructed with 3 latent variables. In both, PCA and PLS-DA models, the best pre-processing for the NMR data was auto-scaling. Thus, we assured not to lose information of any principal metabolite whose peak had low intensity [50].

Evaluation of the model parameters: The assessment of the model was performed using the following parameters: True positive (TP) and negative (TN), sensitivity (Eq. A.1), specificity (Eq. A.2), false positive rate (FPR, Eq. A.3) and negative (FNR), accuracy (Eq. A.4), and efficiency (Eq. A.5). In this research, TP refers to the NMR data of the infected sheep blood sera classified in Class 0 , and FP is used when the healthy control sample is classified erroneously as Class $1[51,52]$.

$$
\begin{aligned}
& \text { Sensitivity }=\frac{T P}{T P+F N} \\
& \text { Specificity }=\frac{T N}{T N+F P}
\end{aligned}
$$




$$
\begin{aligned}
& \text { False Positive Rate }(\mathrm{FPR})=\frac{F P}{T N+F P} \\
& \text { Accuracy }=\frac{T N+T P}{T N+T P+F N+F P} \\
& \text { Efficiency rate }=100-(F P R+F N R)
\end{aligned}
$$

Eq. A.5

\section{Results and Discussion}

Currently, Polymerase Chain Reaction (PCR) is the most reliable method for the detection of Corynebacterium pseudotuberculosis in caseous lesions [53,54]. However, PCR technique requires the isolation and later identification of the bacteria, which leads to an increased exposure to the pathogen. Also, it is impossible to take caseous material from lesions that are located in internal granulomatous lesions from organs such as liver, lungs, and kidneys. Immunodiagnosis assays work well for the identification of the infection with the bacteria in animals presenting CLA clinical signs, but no assay presents $100 \%$ specificity and sensitivity for the CLA diagnosis in infected animals without CLA clinical signs [34]. Therefore, it is very hard to perform a proper diagnosis in caseous lymphadenitis (CLA) subclinical animals that do not present any detectable lesions.

The use of NMR spectroscopy in the search for biomarkers has many advantages, such as high sensitivity and the use of only a small amount of blood from the animal [25,55]. Moreover, the analyst does not have direct contact with the pathogen, a situation that makes this procedure somewhat safer.

${ }^{1} \mathrm{H}$ NMR spectra indicate the presence of many species in the sheep blood serum, for example in $\delta 1.00$ to 4.00 (amino acids, lipids, organic acids, alcohols), $\delta 4.00$ to 5.00 (carbohydrates), and $\delta 6.00$ to 8.00 (aromatic compounds and other) [56]. Examples of ${ }^{1} \mathrm{H}$ NMR spectra recorded for the two sheep blood serum samples are presented in Figure 1a; spectra with the suppression of peaks referring to macromolecules such as peptides, proteins and lipids are shown in Figures $1 \mathrm{~b}$ and $1 \mathrm{c}$ in the spectral regions $\delta 6.50$ to 8.00 and $\delta 0.50$ to 4.20 , respectively.

The spectra presented in Figure 1 have many overlapped broad peaks, typical for a biological sample such as blood serum. Just based on an inspection of the differences among recorded NMR-spectra, it was tough to discriminate the healthy from the infected sera. Subtle profile differences might be observed from the integration values of some peaks (Table S1, Supplementary information); one alternative to solve this problem is to apply chemometrics. Therefore, we used the multivariate classification method Principal Component Analysis (PCA) primarily and then, the Partial Least Squares Discriminant Analysis (PLS-DA) for samples differentiation.

Due to the vast amount of data and to the fact that metabolic changes are sometimes discrete, we have used PCA to preserve the most relevant data without compromising the quality of research. PCA and PLS-DA enabled us to gain insight on the spectral region that suffered the most significant change $[57,58]$ upon infection provoked with the C. pseudotuberculosis.

Scores results of the PCA analysis are shown in Figure 2, where we can observe a tendency to a slight separation into healthy or infected sheep blood serum samples along the PC 2, but close to the Score 0 (PC 2 ), the samples form an overcrowded mix.

The Principal Component Analysis (PCA) was performed using all NMR-data. The $T_{2}$-edited ${ }^{1} \mathrm{H}$-NMR data were not considered in chemometrics, because relaxation times for some low molecular weight
(LMW) molecules may also be affected by the suppression of the peaks of the high molecular weight (HMW) molecules.

The Y predicted values by PLS-DA model are shown in Figure 3. Just two samples, one from the healthy control group and the other from the infected group, were mistakenly classified and were considered as FP and FN, correspondingly. However, there are infected sheep blood sera with prediction values very close to the threshold line. In the validation set, the infected sheep blood sera were also close to the threshold line. Still, only one of these samples was misclassified.

Evaluation of the model parameters was organized in the matrix presented in Table 1. Analyzing the values of sensitivity and specificity obtained in the PLS-DA model in training and test sets, we can conclude that the values were close, which indicate a good concordance between the training and the test sets. In other words; there is no overfitting, and the training set is representative. Another way to evaluate the model is through accuracy and efficiency values, which indicate the quality of the

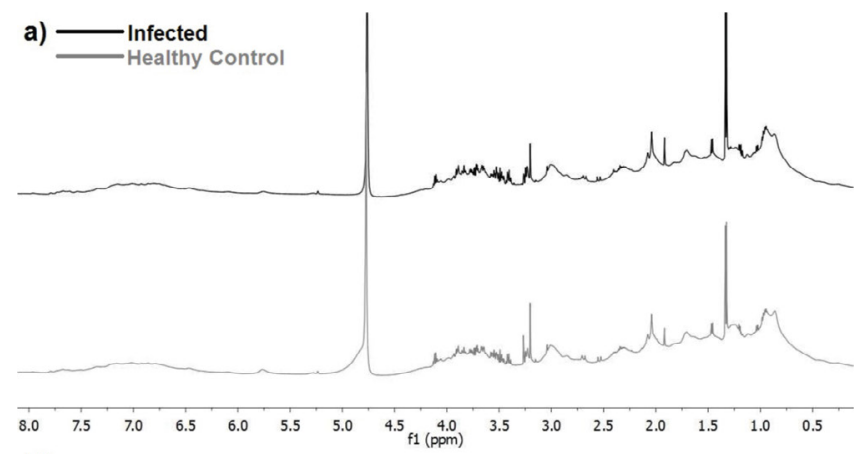

b)
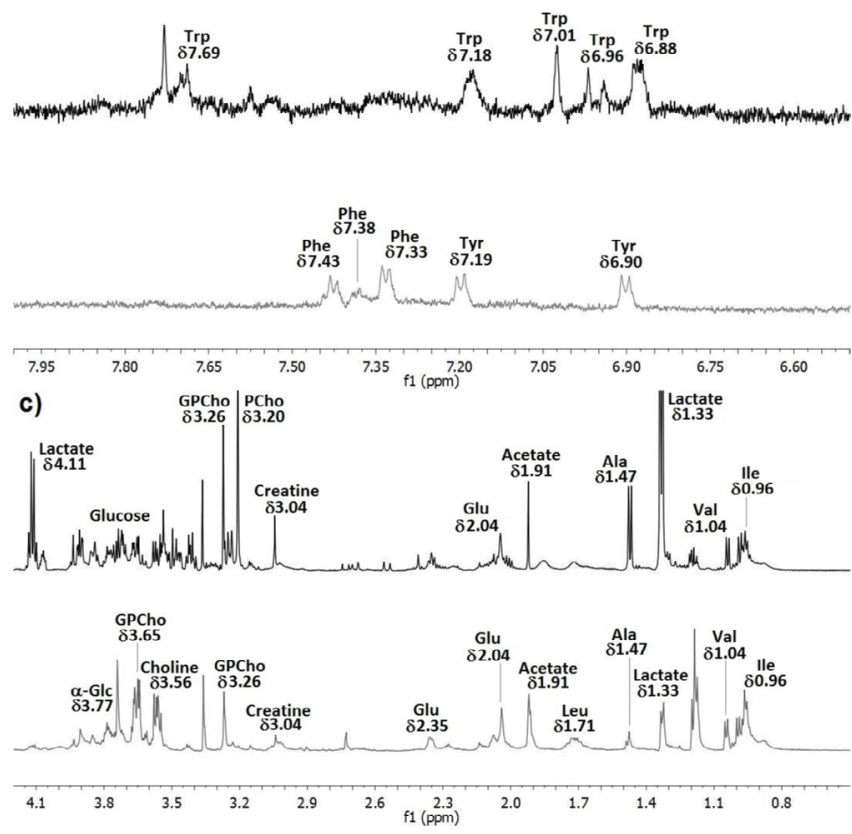

a) ${ }^{1} \mathrm{H}$ NMR spectra obtained from Corynebacterium pseudotuberculosis healthy control sheep (gray) and infected sheep (black) in the spectral region of $\delta 0.00$ to 9.00 ; b) ${ }^{1} \mathrm{H}$ NMR with $T 2$ filter spectra for $\delta 6.50$ to 8.00 ; c) ${ }^{1} \mathrm{H}$ NMR with $T 2$ filter for $\delta 0.50$ to 4.20 . Tryptophan (Trp), phenylalanine (Phe), tyrosine (Tyr), glycerylphosphocholine (GPCho), phosphocholine (PCho), glutamate (Glu), alanine (Ala), valine (Val), isoleucine (lle), alpha-glucose (Glc) and leucine (Leu).

Figure 1: ${ }^{1} \mathrm{H}$ NMR spectra of sheep blood sera. 


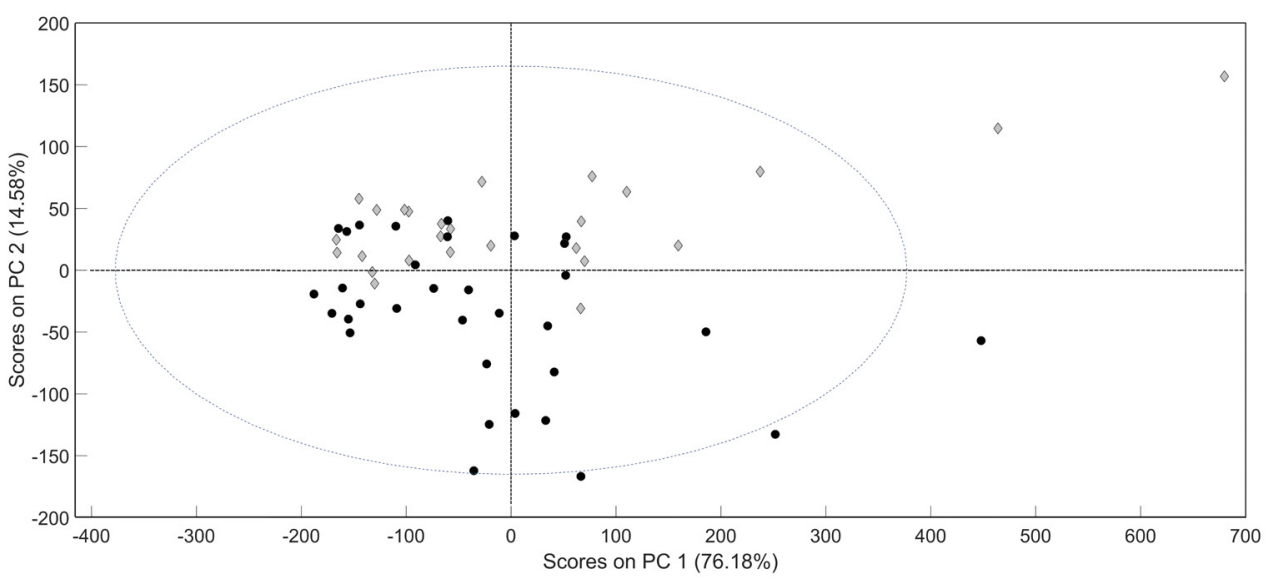

Scores graph PCA (PC $1 \times$ PC 2) for 59 samples, being 33 infected sheep blood sera (black circles) and 26 healthy control samples (gray rhombus).

Figure 2: Scores graph of principal component analysis.

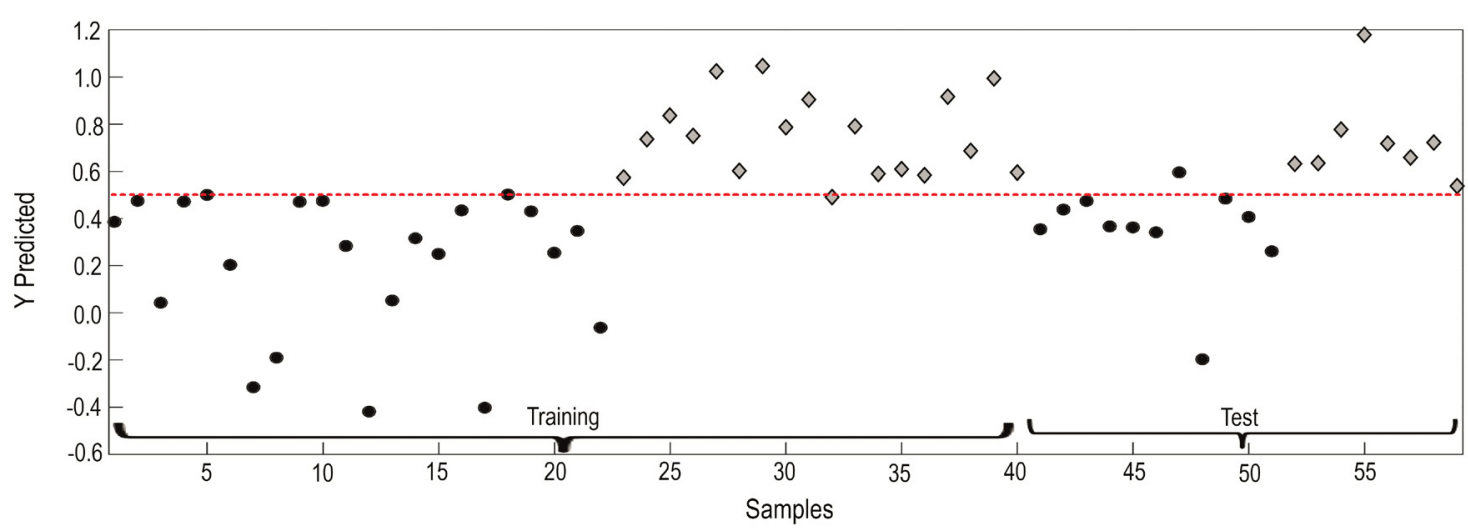

Estimated class values (Y Predicted) for training and test sets, for discrimination between healthy and infected animals by PLS-DA. 33 infected sheep blood sera (black circles); and 26 healthy control samples (gray rhombus) were analyzed. Training set was built from the 40 samples: 22 infected sheep blood sera (0 to 22) and 18 healthy control samples (23 to 40). Validation set (total of 19 samples) used was built from 11 infected sheep blood sera (41 to 51 ) and 8 healthy control samples (52 to 59 ).

Figure 3: Partial least squares discriminant analysis.

\begin{tabular}{|c|c|c|}
\hline Parameters & Training set (\%) & Test set (\%) \\
\hline False Positive Rate (FPR) & 0.00 & 9.09 \\
\hline False Negative Rate (FNR) & 5.56 & 0.00 \\
\hline Sensitivity & 94.44 & 100.00 \\
\hline Specificity & 100.00 & 90.91 \\
\hline Accuracy & 97.50 & 94.74 \\
\hline Efficiency & 94.44 & 90.91 \\
\hline
\end{tabular}

Sensitivity is expressed as the probability for an infected sheep blood serum sample to be classified as Class 0 , and specificity as the probability that a healthy control is wrongly classified in Class 1 . Accuracy is the rate of correct classification, independent of the class of the sample, and efficiency rate is defined as the difference between the total of results (100\%) and the sum of FPR and FNR.

Table 1: Classification parameters obtained for PLS-DA model.

model when assessing two classes simultaneously. These values for the presented PLS-DA model were higher than $90 \%$ in both sets (training and test), thus pointing to an excellent discrimination between healthy and infected sheep blood sera.

Through the PLS-DA developed model, it was possible to identify the variables that are the most important to establish the relationship between matrix X $\left({ }^{1} \mathrm{H}\right.$ NMR spectra profile) and Y (the classes of serum). We can highlight the variable importance in projection (VIP)available in an online resource. The idea behind VIP is to accumulate the importance of each variable $j$ being reflected by the weights from each latent variable [59].

Variables with VIP scores greater than 1 were the most important Chemical Shifts for the discrimination of the two classes of samples in PLS-DA, these variables were highlighted in the mean spectrum of all samples. Thus, NMR spectral regions that contributed to the separation between healthy and infected sheep blood sera were: $\delta-0.20$ to 2.20 ; 3.20 to 4.40 and 6.40 to 8.00 . With the objective to identify the relevant metabolites, we have used the HSQC correlations and online databases such as Biological Magnetic Resonance Data Bank-BMRB [60] and the Platform for Riken Metabolomics-PRIMe [61]. Moreover, twenty metabolites were identified using the cited database, as presented in Table S4 (on-line resource). Also, examples of the HSQC contour maps obtained for the healthy and infected sheep's blood sera, ${ }^{1} \mathrm{H}$ NMR with $T_{2}$ filter and HSQC correlations $\left(\delta_{\mathrm{H}}-\delta_{\mathrm{C}}\right)$-Tables S2 and S3-used in metabolomics database searches are available in an online resource. 
Based on results of peak assignments (Table S5 in the on-line resource) that reflect the differences in concentration or differences in chemical nature, the following metabolites can be cited as the most responsible for two groups separation: L- tryptophan, L-phenylalanine, L-tyrosine, D-(+)-mannose, D-(+)-galactitol, D-(+)-mannitol, L-ornithine, L-leucine, and L-arginine.

It also has been observed an increase of alcohols, such as butanol and sorbitol in the infected sheep blood samples, which can be related to the fragmentation of mycolic acid, the last one is exclusively present in the Corynebacterium genus [62].

Furthermore, a decrease in the intensity of the peaks in the $\delta 3.00$ 4.00 range in infected sheep blood sera (Figure 1c) was observed. It is expected that the decreased levels of sugars, such as $\mathrm{D}-(+)$-glucose and $\mathrm{D}-(+)$-mannose, contributed to this observation. This decrease can be correlated to the fermentation processes that are typical for the biochemical action of C. pseudotuberculosis [63]. However, the greater diversity of the peaks at this spectral region $(\delta 3.00-4.00)$ seen in the infected sheep blood serum spectra can be due to the presence of other C. pseudotuberculosis sugars.

L-Tryptophan was identified only in serum samples from infected (sick) animals (Figure 1b). In general, tryptophan is synthesized in bacteria $[64,65]$. We believe that in the infection process this amino acid might be excreted to the blood. The increase in the blood tryptophan levels is linked to some skin histopathologies, while quinolinate, formed through tryptophan catabolism, was previously reported in lymph nodes [66].

In healthy control samples, we have detected slightly higher amounts of the following amino acids: L-arginine, Ala-Ala, L-leucine, L-ornithine, L-phenylalanine, and L-tyrosine. We might assume that infected animals use these amino acids for the formation of new immune cells via processes of mitosis and expansion, which $\mathrm{T}$ and $\mathrm{B}$ lymphocytes undergo after antigen recognition and activation [67].

C. pseudotuberculosis infected animals usually develop a significant immune response as can be observed through the formation of granulomatous lesions in lymphoid tissues. This bacterium is also known to induce a strong chemotaxis action on many immune cells that will construct the granuloma [68]. Therefore, a marked presence of lipids with the fatty acids' long chains can be explained as a consequence of the production of lipidic intermediates originated from the degradation of the arachidonic acid (phospholipids, PL), which is a well-known feature when inflammation process occurs [69], such in chemotaxis of immune cells.

Negative chemical shifts, resultant of intermolecular interactions [70], very likely indicate variations in the levels of some proteins in serum samples upon infection, such as immunoglobulins. There is no knowledge on immunoglobulins characterization studies by NMR for the CLA. However, there are reports on the improved resistance to infection provoked with the C. pseudotuberculosis in mice [71]. Thus, CLA progress in sheep might be associated with the high levels of haptoglobin and IgM [72].

\section{Conclusion}

Very successfully, a pioneer NMR-based metabolomics research has been applied for the Caseous Lymphadenitis (CLA) biomarkers' identification in sheep. Twenty metabolites were detected as altered in sheep blood serum samples when compared healthy and infected animals. Among those, fourteen figured as possible CLA key- metabolites, 9 found only in the healthy sample group and 5 in the infected sheep blood sera. Their chemical shifts belong to the following NMR-spectral regions: $\delta-0.20$ to $2.20 ; 3.20$ to 4.40 and 6.40 to 8.00 , which showed to have very high loadings in the PCA and PLS-DA. We also have shown that exclusive Corynebacterium pseudotuberculosis metabolites, like alcohols, sugars and tryptophan, could be observed applying NMR with ease. Thus, based on the reported data, we hope that future research might contribute to the development of a safer caseous lymphadenitis diagnosis and/or bring new solutions to immunoprohylaxis of the CLA disease.

\section{Acknowledgments}

We thank the Fundação de Amparo à Pesquisa do Estado de São Paulo (FAPESP, São Paulo, Brazil), Conselho Nacional de Desenvolvimento Científico e Tecnológico (CNPq, Brasília, Brazil) and Coordenação de Aperfeiçoamento de Pessoal de Nível Superior (CAPES, Brasília, Brazil) for grants and fellowships.

\section{Ethics Statement}

We confirm that the specific permit under the Protocol $n^{\circ} .321 / 2015$, related to the Project "Development of a bivalent vaccine against caseous lymphadenitis and toxoplasmosis for small ruminants", under the supervision of Vasco Ariston de Carvalho Azevedo, is in agreement with the Ethical Principles in Animal Experimentation, and adopted by the Ethics Committee in Animal Experimentation (CEUA/UFMG) was required for the described field studies.

The sheep under the study were bred at the Animal Facilities of the Laboratory of Immunology and Molecular Biology (Labimuno-UFBA), located in the municipality

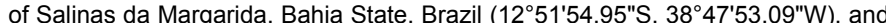
were purchased from the local sheep breeders for the scientific purposes. Animals were fed with grass, protein concentrate and water ad libitum, as recommended by the Animal Nutrition Laboratory of the Federal University of Bahia (UFBA). Collecting of the animal blood serum samples was conducted by veterinarians and followed the recommendations of the Brazilian Ministry of Agriculture (MAPA 2010). After the samples had been obtained, animals were closely observed for a two days period with the objective to detect any lesion or behavioral problems due to the procedures.

We confirm that this manuscript is the result of a basic research project that was developed at the University and that was funded by public funding agencies with the aim of developing new knowledge. The generated results should, therefore, be shared with the scientific and technological community through Open University theses and manuscripts that are published in standard scientific journals. In this sense, we would like to state that we fully adhere to all of the Metabolomics: open access policies regarding the sharing of data and materials. We confirm that this study did not involve endangered or protected species.

\section{Supplementary Information}

The contents of the SI include correlations spectroscopic, HSQC and edited HSQC spectra, $T_{2}$-edited ${ }^{1} \mathrm{H}$-NMR spectra, acquisition parameters, mean integration peaks of ${ }^{1} \mathrm{H}-\mathrm{NMR}$, Variable Importance in Projection (VIP) and details about the 20 metabolites identified (ID and assignments).

\section{References}

1. Varela-Castro L, Lara-Vergara J, Ortega N, Salinas J, Colom-Cadena A, et al. (2017) Endemic caseous lymphadenitis in a wild Caprinae population. Vet Record

2. Oliveira M, Barroco C, Mottola C, Santos R, Lemsaddek A, et al. (2014) First report of Corynebacterium pseudotuberculosis from caseous lymphadenitis lesions in Black Alentejano pig (Sus scrofa domesticus). Vet Res 10: 218.

3. Spier SJ (2008) Corynebacterium pseudotuberculosis infection in horses: An emerging disease associated with climate change? Equine Vet Educ 20: 37-39.

4. Yeruham I, Friedman S, Perl S, Elad D, Berkovich Y, et al. (2004) A herd level analysis of a Corynebacterium pseudotuberculosis outbreak in a dairy cattle herd. Vet Dermatol 15: 315-320.

5. Selim SA (2001) Oedematous skin disease of buffalo in Egypt. J Vet Med Series B 48: 241-258.

6. Morales N, Aldridge D, Bahamonde A, Cerda J, Araya C, et al. (2017) Corynebacterium pseudotuberculosis infection in Patagonian Huemul (Hippocamelus bisulcus). J Wildlife Dis. 
7. Peel MM, Palmer GG, Stacpoole AM, Kerr TG (1997) Human Lymphadenitis due to Corynebacterium pseudotuberculosis: Report of ten cases from Australia and review. Clin Infect Dis 24: 185-191.

8. Heggelund L, Gaustad P, Håvelsrud OE, Blom J, Borgen L, et al. (2015) Corynebacterium pseudotuberculosis Pneumonia in a veterinary student infected during laboratory work. Open Forum Infect Dis 2: ofv053.

9. Paton MW, Rose IR, Hart RA, Sutherland SS, Mercy AR, et al. (1994) New infection with Corynebacterium pseudotuberculosis reduces wool production. Aust Vet J 71: 47-49.

10. Augustine JL, Renshaw HW (1986) Survival of Corynebacterium pseudotuberculosis in axenic purulent exudate on common barnyard fomites. Am J Vet Res 47: 713

11. Washburn K (2016) Caseous Lymphadenitis of sheep and goats.

12. Hassan SS, Guimarães LC, Pereira UP, Islam A, Ali A, et al. (2012) Complete genome sequence of Corynebacterium pseudotuberculosis biovar ovis strain P54B96 isolated from antelope in South Africa obtained by rapid next generation sequencing technology. Standards in Genomic Sciences 7: 189-199.

13. Cerdeira LT, Pinto AC, Schneider MP, de Almeida SS, dos Santos AR, et al. (2011) Whole-genome sequence of Corynebacterium pseudotuberculosis PAT10 strain isolated from sheep in Patagonia, Argentina. J Bacteriol 193 6420-6142.

14. Paton MW, Sutherland SS, Rose IR, Hart FW, Mercy AR, et al. (1995) The spread of Corynebacterium pseudotuberculosis infection to unvaccinated and vaccinated sheep. Aust Vet J 72: 266-269.

15. Guimarães AS, Seyffert N, Bastos BL, Portela RWD, Meyer R, et al. (2009) Caseous lymphadenitis in sheep flocks of the state of Minas Gerais, Brazil: Prevalence and management surveys. Small Ruminant Research 87: 86-91.

16. Kilcoyne I, Spier SJ, Carter CN, Smith JL, Swinford AK, et al. (2014) Frequency of Corynebacterium pseudotuberculosis infection in horses across the United States during a 10-year period. J Am Vet Med Assoc 245: 309-314.

17. Connor KM, Quirie MM, Baird G, Donachie W (2000) Characterization of United Kingdom isolates of Corynebacterium pseudotuberculosis using Pulsed-Field Gel Electrophoresis. J Clin Microbiol 38: 2633.

18. Arsenault J, Girard C, Dubreuil P, Daignault D, Galarneau JR, et al. (2003) Prevalence of and carcass condemnation from maedi-visna, paratuberculosis and caseous lymphadenitis in culled sheep from Quebec, Canada. Prev Vet Med 59: 67-81.

19. Stanford K, Brogden KA, McClelland LA, Kozub GC, Audibert F (1998) The incidence of caseous lymphadenitis in Alberta sheep and assessment of impact by vaccination with commercial and experimental vaccines. Can J Vet Res 62: 38 .

20. Barh D, Jain N, Tiwari S, Parida BP, D'Afonseca V, et al. (2011) A novel comparative genomics analysis for common drug and vaccine targets in Corynebacterium pseudotuberculosis and other CMN group of human pathogens. Chem Biol Drug Design 78: 73-84.

21. http://www.aces.edu/pubs/docs/U/UNP-0085/index2.tmp

22. Fontaine MC, Baird G, Connor KM, Rudge K, Sales J, et al. (2006) Vaccination confers significant protection of sheep against infection with a virulent United Kingdom strain of Corynebacterium pseudotuberculosis. Vaccine 24: 59865996.

23. Soares SC, Trost E, Ramos RTJ, Carneiro AR, Santos AR, et al. (2013) Genome sequence of Corynebacterium pseudotuberculosis biovar equi strain 258 and prediction of antigenic targets to improve biotechnological vaccine production. J Biotechnol 167: 135-141.

24. Sussulini A, Prando A, Maretto DA, Poppi RJ, Tasic L, et al. (2009) Metabolic profiling of human blood serum from treated patients with bipolar disorder employing ${ }^{1} \mathrm{H}$ NMR spectroscopy and chemometrics. Anal Chem 81: 9755-9763.

25. Smolinska A, Blanchet L, Buydens LMC, Wijmenga SS (2012) NMR and pattern recognition methods in metabolomics: From data acquisition to biomarker discovery: A review. Analytica Chimica Acta 750: 82-97.

26. Bharti SK, Roy R (2014) Metabolite identification in NMR-based metabolomics Curr Metabol 2: 163-173.

27. Pontes JGM, Brasil AJM, Cruz GCF, Souza RN, Tasic L (2017) NMR-based metabolomics strategies: plants, animals and humans. Anal Methods 9: 10781096.
28. Pontes JGM, Ohashi WY, Brasil AJM, Filgueiras PR, Espíndola APDM, et al. (2016) Metabolomics by NMR Spectroscopy in Plant Disease diagnostic: Huanglongbing as a Case Study. Chem Select 1: 1176-1178.

29. Wakaskar RR, Bathena SPR, Tallapaka S, Ambardekar VV, Gautum N, et al. (2015) Peripherally cross-linking the shell of core-shell polymer micelles decreases premature release of physically loaded combretastatin A4 in whole blood and increases its mean residence time and subsequent potency against primary murine breast tumors after IV administration. Pharmaceut Res 32 : 1028-1044.

30. Xavier ER, Capanema BP, Ruiz JC, Oliveira G, Meyer R, et al. (2008) Brazilian genome sequencing projects: state of the art. In: Bronner C (ed.) Recent Patents on DNA \& Gene Sequences, Bentham Science Publishers, Sharjah 2: $111-132$.

31. Soares SC, Silva A, Trost E, Blom J, Ramos R, et al. (2013) The pan-genome of the animal pathogen Corynebacterium pseudotuberculosis reveals differences in genome plasticity between the biovar ovis and equi strains. PLoS ONE 8 : e53818.

32. Heydari H, Siow CC, Tan MF, Jakubovics NS, Wee WY, et al. (2014) CoryneBase: Corynebacterium genomic resources and analysis tools at you fingertips. PLoS ONE 9: e86318.

33. Pinto AC, Sá PHCG, Ramos RTJ, Barbosa S, Barbosa HPM, et al. (2014) Differential transcriptional profile of Corynebacterium pseudotuberculosis in response to abiotic stresses. Genomics 15: 14

34. Moura-Costa LF, Bahia RC, Carminati R, Vale VLC, Paule BJA, et al. (2008) Evaluation of the humoral and cellular immune response to different antigens of Corynebacterium pseudotuberculosis in Canindé goats and their potential protection against caseous lymphadenitis. Vet Immunol Immunopathol 126: 131-141.

35. Pacheco LG, Pena RR, Castro TL, Dorella FA, Bahia RC, et al. (2007) Multiplex PCR assay for identification of Corynebacterium pseudotuberculosis from pure cultures and for rapid detection of this pathogen in clinical samples. $J$ Med Microbiol 56: 480-486.

36. Rebouças MF, Loureiro D, Bastos BL, Moura-Costa LF, Hanna SA, et al. (2013) Development of an indirect ELISA to detect Corynebacterium pseudotuberculosis specific antibodies in sheep employing T1 strain culture supernatant as antigen. Pesquisa Veterinária Brasileira 33: 1296-1302.

37. Jue T, Arias-Mendoza F, Gonnela NC, Shulman GI, Shulman RG (1985) A ${ }^{1} \mathrm{H}$ NMR technique for observing metabolite signals in the spectrum of perfused liver. Proc Natl Acad Sci U S A 82: 5246-5249.

38. Meiboom S, Gill D (1958) Modified spin-echo method for measuring nuclear relaxation times. Rev Sci Instrum 29: 688-691.

39. Rinnan Å, Berg F, Engelsen SB (2009) Review of the most common preprocessing techniques for near-infrared spectra. TrAC 28: 1201-1222.

40. Liu H, Tayyari F, Edison AS, Su Z, Gu L (2016) NMR-based metabolomics reveals urinary metabolome modifications in female Sprague-Dawley rats by cranberry procyanidins. J Nutr Biochem 34: 136-145.

41. Robinette SL, Ajredini R, Rasheed H, Zeinomar A, Schroeder FC, et al. (2011) Hierarchical alignment and full resolution pattern recognition of 2D NMR Spectra: Application to nematode chemical ecology. Anal Chem 83: 1649-1657.

42. Savorani F, Tomasi G, Engelsen SB (2010) icoshift: A versatile tool for the rapid alignment of 1D NMR spectra. J Magn Reson 202: 190-202.

43. Wise BM, Gallagher NB, Bro R, Shaver JM, Windig W, et al. (2006) Chemometrics Tutorial for PLS_Toolbox and Solo. Eigenvector Research, Inc. 3905.

44. Barker M, Rayens W (2003) Partial least squares for discrimination. J Chemometr 17: 166-173.

45. Quintás G, Portillo N, Garcıa-Cañaveras JC, Castell JV, Ferrer A, et al. (2012) Chemometric approaches to improve PLSDA model outcome for predicting human non-alcoholic fatty liver disease using UPLC-MS as a metabolic profiling tool. Metabolomics 8: 86-98.

46. Górski Ł, Sordoń W, Ciepiela F, Kubiak WW, Jakubowska M (2016) Voltammetric classification of ciders with PLS-DA. Talanta 146: 231-236.

47. Szymańska E, Saccenti E, Smilde AK, Westerhuis JA (2012) Double-check: validation of diagnostic statistics for PLS-DA models in metabolomics studies. Metabolomics 8: 3-16.

48. Pulido A, Ruisánchez I, Boqué R, Rius FX (2003) Uncertainty of results in routine qualitative analysis. TrAC 22: 647-654. 
Citation: De Moraes Pontes JG, De Santana FB, Portela RW, Azevedo V, Poppi RJ, et al. (2017) Biomarkers of the Caseous Lymphadenitis in Sheep by NMR-Based Metabolomics. Metabolomics (Los Angel) 7: 190. doi:10.4172/2153-0769.1000190

49. Rácz A, Bajusz D, Fodor M, Héberger K (2016) Comparison of classification methods with "n-class" receiver operating characteristic curves: A case study of energy drinks. Chemometrics and Intelligent Laboratory Systems 151: 34-43.

50. van den Berg RA, Hoefsloot HCJ, Westerhuis JA, Smilde AK, van der Wer MJ (2006) Centering, scaling, and transformations: improving the biological information content of metabolomics data. BMC Genomics 7: 142

51. Botelho BG, Reis N, Oliveira LS, Sena MM (2015) Development and analytical validation of a screening method for simultaneous detection of five adulterants in raw milk using mid-infrared spectroscopy and PLS-DA. Food Chem 181: 31-37.

52. Santana FB de, Gontijo LC, Mitsutake H, Mazivila SJ, Souza LM de, et al. (2016) Non-destructive fraud detection in rosehip oil by MIR spectroscopy and chemometrics. Food Chem 209: 228-233.

53. Cetinkaya B, Karahan M, Atil E, Kalin R, De Baere T, et al. (2002) Identification of Corynebacterium pseudotuberculosis isolates from sheep and goats by PCR. Vet Microbiol 88: 75-83

54. Kumar J, Tripathi BN, Kumar R, Sonawane GG, Dixit SK (2013) Rapid detection of Corynebacterium pseudotuberculosis in clinical samples from sheep. Trop Anim Health Prod 45: 1429-1435.

55. Pontes JGM, Brasil AJM, Cruz GCF, Souza RN, Tasic L (2017) ${ }^{1} \mathrm{H}$ NMR metabolomic profiling of human and animal blood serum samples. Methods Mol Biol 1546: 275-282.

56. Pimenta LPS, Kim HK, Verpoorte R, Choi YH (2013) NMR-based metabolomics: A probe to utilize biodiversity. In: Roessner U, Dias DA (eds.) Tools for natural product discovery: Methods and protocols, methods in molecular biology, Springer Science+Business Media, Germany, pp: 117-127.

57. Sharaf MA, Illman DL, Kowalski BR (1986) Chemometrics. John Wiley \& Sons NY, USA.

58. Wold S, Esbensen K, Geladi P (1987) Principal Component Analysis. C Chemometr Intell Lab Syst 2: 37-52.

59. Mehmood T, Liland KH, Snipen L, Sæbø S (2012) Chemometrics and Intelligent Laboratory Systems A review of variable selection methods in Partial Least Squares Regression. Chemometr Intell Lab Syst 118: 62-69.

60. Ulrich EL, Akutsu H, Doreleijers JF, Harano Y, loannidis YE, et al. (2008) BioMagResBank. Nucl Acid Res 36: D402-D408.
61. Sakurai T, Yamada Y, Sawada Y, Matsuda F, Akiyama K, et al. (2013) PRIMe Update: innovative content for plant metabolomics and integration of gene expression and metabolite accumulation. Plant Cell Physiol 54: e5.

62. Ioneda T (1993) Chromatographic and mass spectrometric characterization of 3-o-benzoyl methyl ester derivatives of mycolic acid fractions from Corynebacterium pseudotuberculosis, C. diphtheriae and Rhodococcus rhodochrous. Chem Phys Lipids 65: 93-101.

63. Bastos BL, Portela RWD, Dorella FA, Ribeiro D, Seyffert N, et al. (2012) Corynebacterium pseudotuberculosis: Immunological responses in animal models and zoonotic potential. J Clin Cell Immunol S4: 005.

64. http://www.genome.jp/kegg-bin/show_pathway?cpu00380

65. Silva WM, Carvalho RD, Soares SC, Bastos IFS, Folador EL, et al. (2014) Labelfree proteomic analysis to confirm the predicted proteome of Corynebacterium pseudotuberculosis under nitrosative stress mediated by nitric oxide. Bio Med Central Genomics 15: 1065.

66. Moffett JR, Namboodiri A (2003) Tryptophan and the immune response. Immunol Cell Biol 81: 247-265.

67. Abbas AK, Lichtmann AHH, Pillai S (2014) Cellular and Molecular Immunology $\left(8^{\text {th }}\right.$ edn. $)$. Saunders, Philadelphia

68. Pépin M, Pittet JC, Olivier M, Gohin I (1994) Cellular composition of Corynebacterium pseudotuberculosis pyogranulomas in sheep. J Leukocyte Biol 56: 666 .

69. Buckley CD, Gilroy DW, Serhan CN (2014) Proresolving lipid mediators and mechanisms in the resolution of acute inflammation. Immunity 40: 315-327.

70. Avbelj F, Kocjan D, Baldwin RL (2004) Protein chemical shifts arising from $\alpha$-helices and $\beta$-sheets depend on solvent exposure. Proc Natl Acad Sci U S A 101: 17394-17397.

71. Irwin MR, Knight HD (1975) Enhanced resistance to Corynebacterium pseudotuberculosis infections associated with reduced serum immunoglobulin levels in levamisole-treated mice. Infect Immun 12: 1098.

72. Bastos BL, Loureiro D, Raynal JT, Guedes MT, Costa Vale VL, et al. (2013) Association between haptoglobin and IgM levels and the clinical progression of caseous lymphadenitis in sheep. BMC Vet Res 9: 254 